\title{
Approximate Solution of Non-Linear Fractional Klein-Gordon Equation Using Spectral Collocation Method
}

\author{
Rubayyi T. Alqahtani \\ Department of Mathematics and Statistics, College of Science, Al-Imam Mohammad Ibn Saud Islamic \\ University (IMSIU), Riyadh, Saudi Arabia \\ Email: rtalqahtani@imamu.edu.sa
}

Received 1 September 2015; accepted 27 November 2015; published 30 November 2015

Copyright (C) 2015 by author and Scientific Research Publishing Inc.

This work is licensed under the Creative Commons Attribution International License (CC BY). http://creativecommons.org/licenses/by/4.0/

c) (i) Open Access

\begin{abstract}
In this paper, we implement the spectral collocation method with the help of the Legendre polynomials for solving the non-linear Fractional (Caputo sense) Klein-Gordon Equation (FKGE). We present an approximate formula of the fractional derivative. The Legendre collocation method is used to reduce FKGE to the solution of system of ODEs which is solved by using finite difference method. The results of applying the proposed method to the non-linear FKGE show the simplicity and the efficiency of the proposed method.
\end{abstract}

\section{Keywords}

Fractional Klein-Gordon Equation, Legendre Spectral Method

\section{Introduction}

The theory of fractional calculus is initiated by Leibniz, Liouville, Riemann, Grunwald and Letnikov and since then has been found many applications in science and engineering. Finding accurate and efficient method for solving fractional differential equations has been an active research subject. Finding the exact solution for most of these equations is not an easy task, thus analytical and numerical methods must be used.

The Klein-Gordon equation plays a significant role in mathematical physics and many scientific applications such as solid-state physics, nonlinear optics, and quantum field theory [1]. The equation has attracted much attention in studying solitons and condensed matter physics, in investigating the interaction of solitons in a collisionless plasma and the recurrence of initial states, and in examining the nonlinear wave equations [2]. Wazwaz has obtained the various exact traveling wave solutions such as compactons, solitons and periodic solutions by 
using the tanh method [1]. The study of numerical solutions of the Klein-Gordon equation has been investigated considerably in the last few years. In the previous studies, the most papers have carried out different spatial discretization of the equation ([2] [3]).

In this work, we apply spectral collocation method (with the help of Legendre polynomials) to obtain the numerical solution of the non-linear FKGE of the form

$$
u_{t t}(x, t)+a D^{\alpha} u(x, t)+b u(x, t)+c u^{\gamma}(x, t)=f(x, t), x \in(0, L), t>0, \alpha \in(1,2],
$$

where $D^{\alpha}$ denotes the Caputo fractional derivative of order $\alpha$ with respect to $x, u(x, t)$ is unknown function, and $a<0, b, c$ and $\gamma$ are known constants with $\gamma \in \mathbb{R}, \gamma \neq \pm 1$.

We consider the initial conditions and the boundary conditions as follows:

$$
\begin{gathered}
u(x, 0)=g_{1}(x), \quad u_{t}(x, 0)=g_{2}(x), \quad x \in(0,1), \\
u(0, t)=u(1, t)=0 .
\end{gathered}
$$

The existence and the uniqueness of the solution of Equations (1)-(3) are given in ([2]-[4]).

For more details about the fractional calculus see ([5]-[8]) and for more details about the Legendre collocation method see ([9]-[18]).

\section{An Approximate Formula of the Fractional Derivative}

The well-known Legendre polynomials are defined on the interval $[-1,1]$ and can be determined with the aid of the following recurrence formula [19]

$$
L_{k+1}(z)=\frac{2 k+1}{k+1} z L_{k}(z)-\frac{k}{k+1} L_{k-1}(z), \quad k=1,2, \cdots
$$

In order to use these polynomials on the interval $[0,1]$ we define the so called shifted Legendre polynomials by introducing the change of variable $z=2 x-1$. Let the shifted Legendre polynomials $L_{k}(2 x-1)$ be denoted by $P_{k}(x)$. Then $P_{k}(x)$ can be obtained as follows:

$$
P_{k+1}(x)=\frac{(2 k+1)(2 x-1)}{(k+1)} P_{k}(x)-\frac{k}{k+1} P_{k-1}(x), \quad k=1,2, \cdots,
$$

where $P_{0}(x)=1$ and $P_{1}(x)=2 x-1$. The analytic form of the shifted Legendre polynomials $P_{k}(x)$ of degree $k$ is given by

$$
P_{k}(x)=\sum_{i=0}^{k}(-1)^{k+i} \frac{(k+i) !}{(k-i)(i !)^{2}} x^{i} .
$$

The function $u(x)$, which is square integrable in $[0,1]$, may be expressed in terms of shifted Legendre polynomials as

$$
u(x)=\sum_{i=0}^{\infty} c_{i} P_{i}(x)
$$

where the coefficients $c_{i}$ are given by

$$
c_{i}=(2 i+1) \int_{0}^{1} u(x) P_{i}(x) \mathrm{d} x, \quad i=1,2, \cdots
$$

In practice, only the first $(m+1)$-terms of shifted Legendre polynomials are considered. Then we have

$$
u_{m}(x)=\sum_{i=0}^{m} c_{i} P_{i}(x)
$$

Theorem 1 [14].

Let $u(x)$ be approximated by shifted Legendre polynomials as (8) and also suppose $\alpha>0$ then 


$$
D^{\alpha}\left(u_{m}(x)\right)=\sum_{i=\lceil\alpha\rceil k=\lceil\alpha\rceil}^{m} \sum_{i}^{i} c_{i} w_{i, k}^{(\alpha)} x^{k-\alpha}, \quad w_{i, k}^{(\alpha)}=\frac{(-1)^{(i+k)}(i+k) !}{(i-k) !(k) ! \Gamma(k+1-\alpha)} .
$$

Theorem 2 [14].

The error $\left|E_{T}(m)\right|=\left|D^{\alpha} u(x)-D^{\alpha} u_{m}(x)\right|$ in approximating $D^{\alpha} u(x)$ by $D^{\alpha} u_{m}(x)$ is bounded by

$$
\left|E_{T}(m)\right| \leq\left|\sum_{i=m+1}^{\infty} c_{i}\left(\sum_{k=\lceil\alpha\rceil}^{i} \sum_{j=0}^{k-\lceil\alpha\rceil} \Theta_{i, j, k}\right)\right|,
$$

where

$$
\Theta_{i, j, k}=\frac{(-1)^{i+k}(i+k) !(2 j+1)}{(i-k) !(k) ! \Gamma(k-\alpha+1)} \times \sum_{r=0}^{j} \frac{(-1)^{j+r}(j+r) !}{(j-r) !(r !)^{2}(k-\alpha+r+1)}, \quad j=0,1, \cdots
$$

\section{Numerical Implementation}

\section{Example 1.}

Consider the fractional-order cubically nonlinear Klein-Gordon problem

$$
u_{t t}(x, t)-D^{\alpha} u(x, t)+u^{3}(x, t)=f(x, t), \quad x \in(0,1), t>0, \alpha \in(1,2],
$$

with the initial and boundary conditions $u(x, 0)=0, u_{t}(x, 0)=0, u(0, t)=0, u(1, t)=t^{2}$, where the source term $f(x, t)$ is given by $f(x, t)=2 x^{\alpha}-\Gamma(\alpha+1) t^{2}+x^{3 \alpha} t^{6}$.

The exact solution of this problem is $u(x, t)=x^{\alpha} t^{2}$.

In order to use the proposed method, we approximate $u(x, t)$ with $m=3$ as

$$
u_{3}(x, t)=\sum_{i=0}^{3} u_{i}(t) P_{i}(x) .
$$

From Equation (11) and Theorem 1 we have

$$
\sum_{i=0}^{3} \ddot{u}_{i}(t) P_{i}(x)-\sum_{i=\lceil\alpha\rceil k=\lceil\alpha\rceil}^{3} \sum_{i}^{i} u_{i}(t) w_{i, k}^{(\alpha)} x^{k-\alpha}+\left(\sum_{i=0}^{3} u_{i}(t) P_{i}(x)\right)^{3}=f(x, t) .
$$

We now collocate Equation (13) at $(m+1-\lceil\alpha\rceil)$ points $x_{p}, \quad p=0,1, \cdots, m-\lceil\alpha\rceil$ as

$$
\sum_{i=0}^{3} \ddot{u}_{i}(t) P_{i}\left(x_{p}\right)-\sum_{i=\lceil\alpha\rceil k=\lceil\alpha\rceil}^{3} \sum_{i}^{i} u_{i}(t) w_{i, k}^{(\alpha)} x_{p}^{k-\alpha}+\left(\sum_{i=0}^{3} u_{i}(t) P_{i}\left(x_{p}\right)\right)^{3}=f\left(x_{p}, t\right) .
$$

For suitable collocation points we use roots of shifted Legendre polynomial $P_{m+1-\lceil\alpha\rceil}(x)$.

In this case, the roots $x_{p}$ of shifted Legendre polynomial $P_{2}(x)$, i.e. $x_{0}=0.1465, x_{1}=0.8873$.

Also, by substituting Equation (12) in the boundary conditions we can find

$$
\sum_{i=0}^{3}(-1)^{i} u_{i}(t)=0, \quad \sum_{i=0}^{3} u_{i}(t)=t^{2}
$$

By using Equation (14) and Equation (15) we obtain the following non-linear system of ODEs:

$$
\begin{gathered}
\ddot{u}_{0}(t)+k_{1} \ddot{1}_{1}(t)+k_{2} \ddot{u}_{3}(t)=\sum_{i=\lceil\alpha\rceil k=\lceil\alpha\rceil}^{3} \sum_{i}^{i} u_{i}(t) w_{i, k}^{(\alpha)} x_{0}^{k-\alpha}-\left(\sum_{i=0}^{3} u_{i}(t) P_{i}\left(x_{0}\right)\right)^{3}+f_{0}(t), \\
\ddot{u}_{0}(t)+k_{11} \ddot{u}_{1}(t)+k_{22} \ddot{u}_{3}(t)=\sum_{i=\lceil\alpha\rceil k=\lceil\alpha\rceil}^{3} \sum_{i}^{i} u_{i}(t) w_{i, k}^{(\alpha)} x_{1}^{k-\alpha}-\left(\sum_{i=0}^{3} u_{i}(t) P_{i}\left(x_{1}\right)\right)^{3}+f_{1}(t), \\
u_{0}(t)-u_{1}(t)+u_{2}(t)-u_{3}(t)=0,
\end{gathered}
$$




$$
u_{0}(t)+u_{1}(t)+u_{2}(t)+u_{3}(t)=t^{2}
$$

where $k_{1}=P_{1}\left(x_{0}\right), k_{2}=P_{3}\left(x_{0}\right), k_{11}=P_{1}\left(x_{1}\right), k_{22}=P_{3}\left(x_{1}\right)$.

Now, to use FDM [20] for solving the system (16)-(19), we will use the following notations: $t_{n}=n \Delta t$ to be the integration time $0 \leq t_{n} \leq T, \Delta t=T / N$, for $n=0,1, \cdots, N$. Define $u_{i}^{n}=u_{i}\left(t_{n}\right), f_{i}^{n}=f_{i}\left(t_{n}\right), i=1,2$. Then the system (16)-(19), is discretized and takes the following form:

$$
\begin{aligned}
& \frac{u_{0}^{n+1}-2 u_{0}^{n}+u_{0}^{n-1}}{\Delta t^{2}}+k_{1} \frac{u_{1}^{n+1}-2 u_{1}^{n}+u_{1}^{n-1}}{\Delta t^{2}}+k_{2} \frac{u_{3}^{n+1}-2 u_{3}^{n}+u_{3}^{n-1}}{\Delta t^{2}} \\
& =\sum_{i=\lceil\alpha\rceil k=\lceil\alpha\rceil}^{3} \sum_{i}^{i} u_{i, k}^{n+1} w_{i, k}^{(\alpha)} x_{0}^{k-\alpha}-\left(\sum_{i=0}^{3} u_{i}^{n+1} P_{i}\left(x_{0}\right)\right)^{3}+f_{0}^{n+1}, \\
& \frac{u_{0}^{n+1}-2 u_{0}^{n}+u_{0}^{n-1}}{\Delta t^{2}}+k_{11} \frac{u_{1}^{n+1}-2 u_{1}^{n}+u_{1}^{n-1}}{\Delta t^{2}}+k_{22} \frac{u_{3}^{n+1}-2 u_{3}^{n}+u_{3}^{n-1}}{\Delta t^{2}} \\
& =\sum_{i=\lceil\alpha\rceil k=\lceil\alpha\rceil}^{3} \sum_{i}^{i} u_{i}^{n+1} w_{i, k}^{(\alpha)} x_{1}^{k-\alpha}-\left(\sum_{i=0}^{3} u_{i}^{n+1} P_{i}\left(x_{1}\right)\right)^{3}+f_{1}^{n+1}, \\
& u_{0}^{n+1}-u_{1}^{n+1}+u_{2}^{n+1}-u_{3}^{n+1}=0, \\
& u_{0}^{n+1}+u_{1}^{n+1}+u_{2}^{n+1}+u_{3}^{n+1}=(n \Delta t)^{2} .
\end{aligned}
$$

This system presents the numerical scheme of the proposed problem and is non-linear system of algebraic equations, and by solving this system yields the numerical solution of the non-linear FKGE (11).

The obtained numerical results by means of the proposed method are shown in Table 1 and Figure 1 and Figure 2. In Table 1, the absolute errors between the exact solution $u_{e x}$ and the approximate solution $u_{a p p r o x}$, at $m=3, m=5$ with the final time $t=1$ are given. But, in Figure 1, we presented comparison between the solution and the approximate solution using the proposed method at $\alpha=1.8$ for different values of the final time $t=0.25$ and 0.5 at time step $\Delta t=0.005$ with $m=3$. Also, in Figure 2, we presented the behavior approximate of solution at $t=2$ for different values of $\alpha=1.1,1.4,1.7$ and 2 at time step $\Delta t=0.005$ with $m=3$.

Example 2.

Consider the fractional cubically non-linear Klein-Gordon problem:

$$
\frac{\partial^{2} u(x, t)}{\partial t^{2}}=D^{\alpha} u(x, t)-\frac{3}{4} u(x, t)+\frac{3}{2} u^{3}(x, t)+f(x, t), 0<x<5, t>0, \alpha \in(1,2],
$$

with the following initial and boundary conditions:

Table 1. The absolute error between the exact and approximate solutions at $m=3$, and $m=5$ with $t=1$.

\begin{tabular}{ccc}
\hline$x$ & $\left|u_{\text {ex }}-u_{\text {oppox }}\right|$ at $m=3$ & $\left|u_{\text {ex }}-u_{\text {opprox }}\right|$ at $m=5$ \\
\hline 0.0 & $0.159675 \mathrm{e}-03$ & $0.741852 \mathrm{e}-04$ \\
0.1 & $0.012354 \mathrm{e}-03$ & $0.852951 \mathrm{e}-04$ \\
0.2 & $0.123654 \mathrm{e}-03$ & $0.756980 \mathrm{e}-04$ \\
0.3 & $0.159753 \mathrm{e}-03$ & $0.753648 \mathrm{e}-04$ \\
0.4 & $0.987123 \mathrm{e}-03$ & $0.820135 \mathrm{e}-04$ \\
0.5 & $0.147852 \mathrm{e}-03$ & $0.954378 \mathrm{e}-04$ \\
0.6 & $0.963852 \mathrm{e}-03$ & $0.942315 \mathrm{e}-04$ \\
0.7 & $0.012345 \mathrm{e}-03$ & $0.654852 \mathrm{e}-04$ \\
0.8 & $0.895423 \mathrm{e}-03$ & $0.023154 \mathrm{e}-04$ \\
0.9 & $0.852347 \mathrm{e}-03$ & $0.0321546 \mathrm{e}-04$ \\
1.0 & $0.123789 \mathrm{e}-03$ & $0.6584231 \mathrm{e}-04$ \\
\hline
\end{tabular}




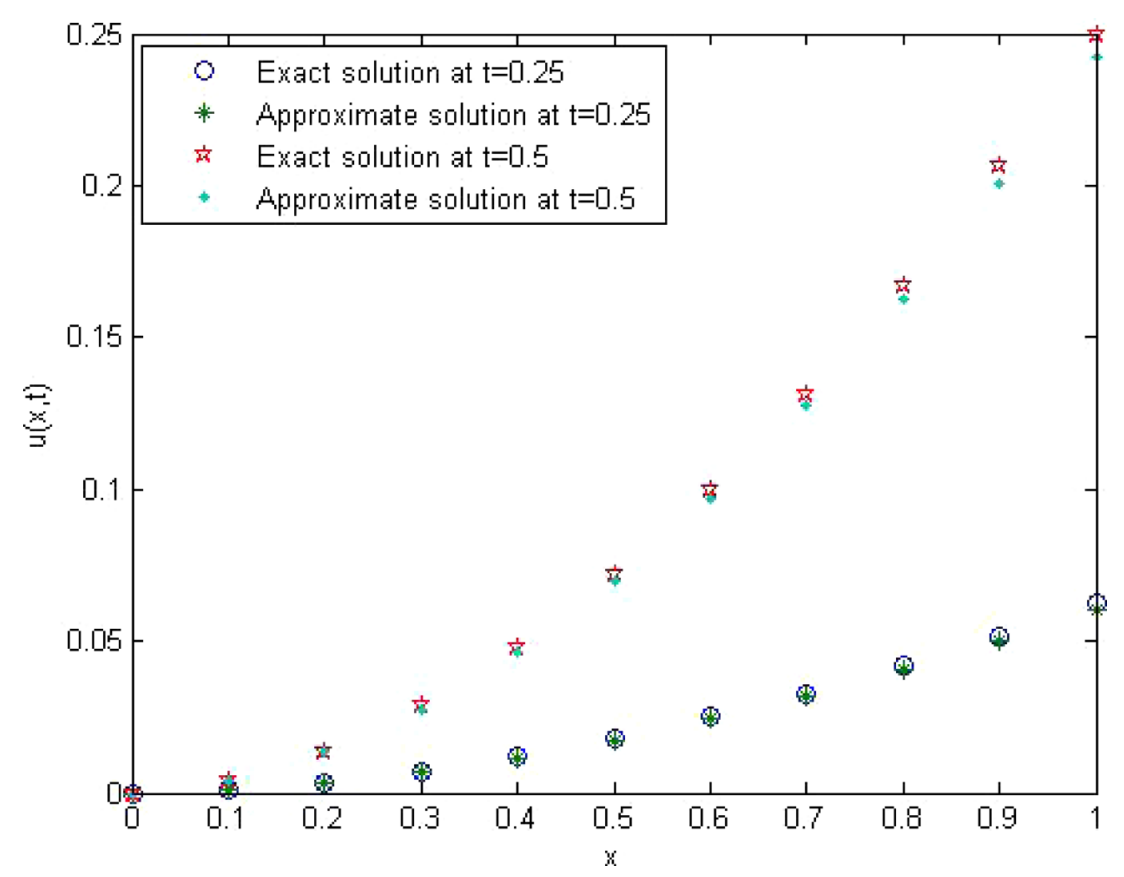

Figure 1. The exact and approximate solutions at $\alpha=1.8$ for different values of $t$.

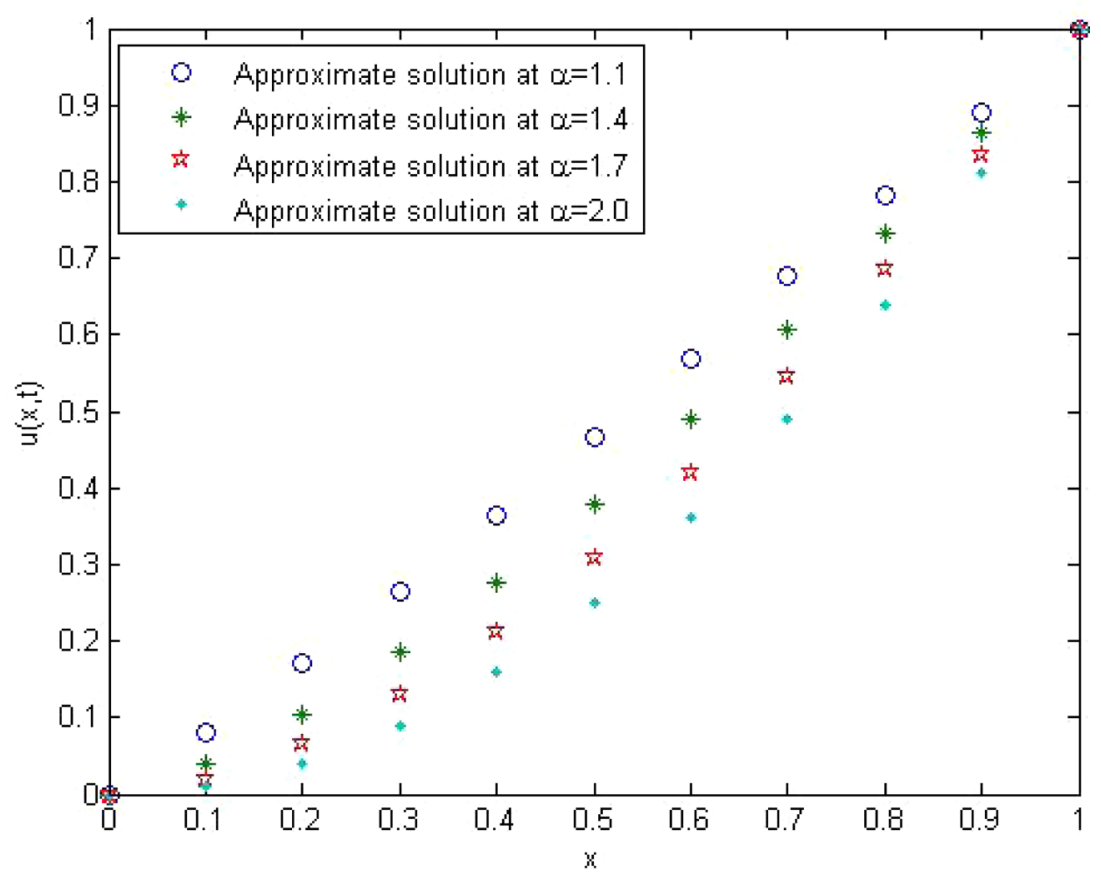

Figure 2. The approximate solution at $t=2$ for different values of $\alpha$.

$$
\begin{array}{ll}
u(x, 0)=0, & u_{t}(x, 0)=0, \\
u(0, t)=0, & u(5, t)=0,
\end{array}
$$

where the source term $f(x, t)$ is given by

$$
f(x, t)=6 x^{\alpha}(5-x) t+x^{\alpha}(5-x) t^{3}-1.5 x^{3 \alpha}(5-x)^{3} t^{9}-(5 \alpha !-(\alpha+1) ! x) .
$$


The exact solution of this problem is $u(x, t)=x^{\alpha}(5-x) t^{3}$.

The obtained numerical results by means of the proposed method are shown in Figure 3 and Figure 4. In Figure 3, we presented comparison between the exact solution and the approximate solution using the proposed method at $\alpha=1.6$ for different values of the final time $t=1.75$ and 1.25 at time step $\Delta=0.005$ with $m=3$. Also, in Figure 4, we presented the behavior of the approximate solution at $t=2$ for different values of $\alpha=1.2,1.5,1.8$ and 2 at time step $\Delta=0.002$ with $m=3$.

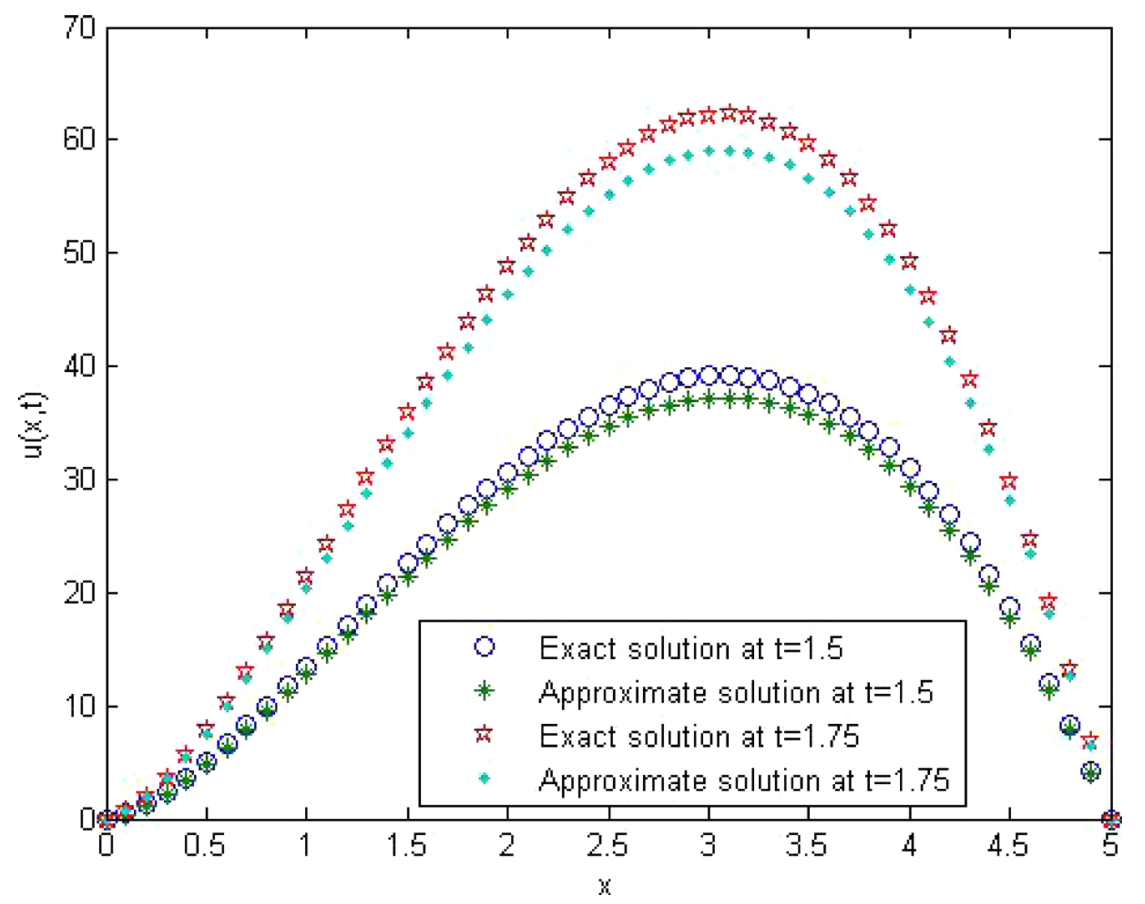

Figure 3. The exact and approximate solutions at $\alpha=1.6$ for different values of time $t$.

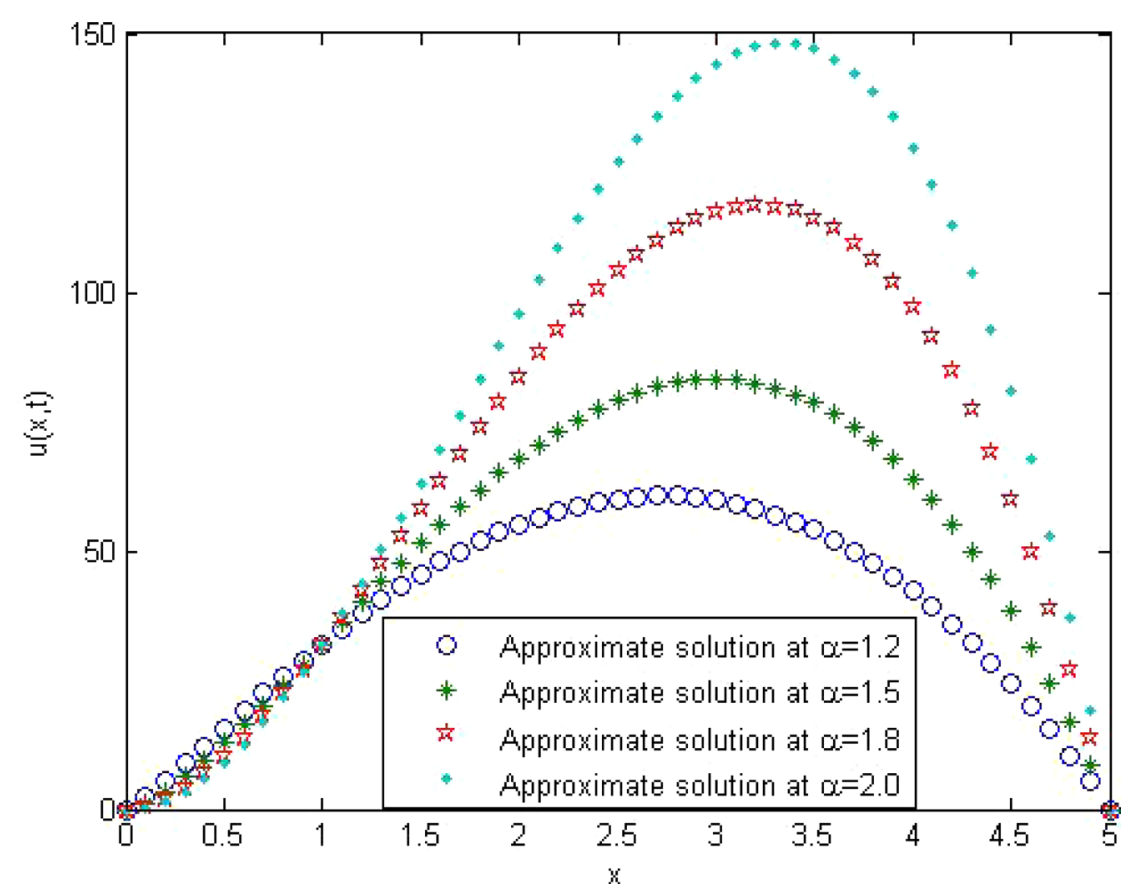

Figure 4. The approximate solution at $t=2$ for different values of $\alpha$. 


\section{Conclusion and Remarks}

We have implemented Legendre spectral method for solving the non-linear FKGE. The proposed method gives excellent results when it is applied to FKGE. Absolute error by the method decreases while increasing iterations or level of resolution or both, as shown in Figures 1-4. It is evident that the overall errors can be made smaller by adding new terms from the series (8). Comparisons are made between approximate solutions and exact solutions to illustrate the validity and the great potential of the technique.

\section{Acknowledgements}

We thank the editor and the referee for their comments.

\section{References}

[1] Wazwaz, A.M. (2006) Compacton Solitons and Periodic Solutions for Some Forms of Nonlinear Klein-Gordon Equations. Chaos, Solitons and Fractals, 28, 1005-1013. http://dx.doi.org/10.1016/j.chaos.2005.08.145

[2] El-Sayed, S.M. (2003) The Decomposition Method for Studying the Klein-Gordon Equation. Chaos, Solitons and Fractals, 18, 1026-1030. http://dx.doi.org/10.1016/S0960-0779(02)00647-1

[3] Yusufoglu, E. (2008) The Variational Iteration Method for Studying the Klein-Gordon Equation. Applied Mathematics Letters, 21, 669-674. http://dx.doi.org/10.1016/j.aml.2007.07.023

[4] El-Sayed, A.M.A., Elsaid, A. and Hammad, D. (2012) A Reliable Treatment of Homotopy Perturbation Method for Solving the Nonlinear Klein-Gordon Equation of Arbitrary (Fractional) Orders. Journal of Applied Mathematics, 2012, 1-13. http://dx.doi.org/10.1155/2012/581481

[5] Doha, E.H., Bhrawy, A.H. and Ezz-Eldien, S.S. (2011) A Chebyshev Spectral Method Based on Operational Matrix for Initial and Boundary Value Problems of Fractional Order. Computers and Mathematics with Applications, 62, 23642373. http://dx.doi.org/10.1016/j.camwa.2011.07.024

[6] El-Sayed, A.M.A., Elsaid, A., El-Kalla, I.L. and Hammad, D. (2012) A Homotopy Perturbation Technique for Solving Partial Differential Equations of Fractional Order in Finite Domains. Applied Mathematics and Computation, 218, 8329-8340. http://dx.doi.org/10.1016/j.amc.2012.01.057

[7] Oldham, K.B. and Spanier, J. (1974) The Fractional Calculus. Academic Press, New York.

[8] Podlubny, I. (1999) Fractional Differential Equations. Academic Press, New York.

[9] Khader, M.M. (2011) On the Numerical Solutions for the Fractional Diffusion Equation. Communications in Nonlinear Science and Numerical Simulations, 16, 2535-2542. http://dx.doi.org/10.1016/j.cnsns.2010.09.007

[10] Khader, M.M. (2015) An Efficient Approximate Method for Solving Fractional Variational Problems. Applied Mathematical Modelling, 39, 1643-1649. http://dx.doi.org/10.1016/j.apm.2014.09.012

[11] Khader, M.M. (2015) Fractional Chebyshev Finite Difference Method for Solving the Fractional-Order Delay BVPs. International Journal of Computational Methods, 12, Article ID: 1550033. http://dx.doi.org/10.1142/s0219876215500334

[12] Khader, M.M. (2014) On the Numerical Solution and Convergence Study for System of Non-Linear Fractional Diffusion Equations. Canadian Journal of Physics, 92, 1658-1666. http://dx.doi.org/10.1139/cjp-2013-0464

[13] Khader, M.M., Sweilam, N.H. and Mahdy, A.M.S. (2011) An Efficient Numerical Method for Solving the Fractional Diffusion Equation. Journal of Applied Mathematics and Bioinformatics, 1, 1-12.

[14] Khader, M.M. and Hendy, A.S. (2012) The Approximate and Exact Solutions of the Fractional-Order Delay Differential Equations Using Legendre Pseudo-Spectral Method. International Journal of Pure and Applied Mathematics, 74, 287-297.

[15] Sweilam, N.H., Khader, M.M. and Al-Bar, R.F. (2007) Numerical Studies for a Multi-Order Fractional Differential Equation. Physics Letters A, 371, 26-33. http://dx.doi.org/10.1016/j.physleta.2007.06.016

[16] Sweilam, N.H. and Khader, M.M. (2010) A Chebyshev Pseudo-Spectral Method for Solving Fractional Integro-Differential Equations. ANZIAM, 51, 464-475. http://dx.doi.org/10.1017/S1446181110000830

[17] Sweilam, N.H., Khader, M.M. and Nagy, A.M. (2011) Numerical Solution of Two-Sided Space-Fractional Wave Equation Using Finite Difference Method. Journal of Computational and Applied Mathematics, 235, 2832-2841. http://dx.doi.org/10.1016/j.cam.2010.12.002

[18] Sweilam, N.H., Khader, M.M. and Mahdy, A.M.S. (2012) Crank-Nicolson Finite Difference Method for Solving Time-Fractional Diffusion Equation. Journal of Fractional Calculus and Applications, 2, 1-9.

[19] Bell, W.W. (1968) Special Functions for Scientists and Engineers. Great Britain, Butler and Tanner Ltd, Frome and London.

[20] Smith, G.D. (1965) Numerical Solution of Partial Differential Equations. Oxford University Press, Oxford. 